\title{
Perceived Treatment Effectiveness, Medication Compliance, and Complementary and Alternative Medicine Use Among Veterans with Bipolar Disorder
}

\author{
Christopher N. Jarman, M.S.W.,', Brian E. Perron, Ph.D., L.C.S.W., \\ Amy M. Kilbourne, Ph.D., M.P.H., ${ }^{2}$ and Carrie Farmer Teh, Ph.D. ${ }^{3}$
}

\begin{abstract}
Objectives: Recent research shows a high rate of complementary and alternative medicine (CAM) use among persons with mental disorders, although correlates and patterns of CAM use are relatively unknown. This study tested whether CAM use is associated with perceived effectiveness of conventional treatment (i.e., psychotropic medication and psychotherapy) and medication compliance among persons with bipolar disorder.

Design: Patients with bipolar disorder $(n=435)$ were included as part of a naturalistic cohort study. Measures of CAM utilization, medication compliance, and perceptions of the effectiveness of psychotropic medications and psychotherapy were based on previously established questionnaires. Associations were tested using bivariate and multivariate analyses.

Results: Bivariate analyses showed that patients who did not perceive psychotherapy as effective at improving social, family, or job functioning reported greater CAM use. However, medication compliance was not significantly associated with use of CAM. Patients who used oral (e.g., herbal therapies) or cognitive (e.g., meditation) CAM were more likely to report that their medications were not effective at relieving manic or depressive symptoms. Users of cognitive CAM were more likely to report that their medications did not help with social, job, or family functioning, and that they did not prevent recurrences of manic or depressive episodes. None of the bivariate associations remained significant in multivariate analyses.

Conclusions: Prior research has suggested that persons who are dissatisfied with treatment for medical conditions are more likely to use CAM therapies. However, the results of this study do not show CAM therapies to be associated with perceived effectiveness of treatments for mental health problems among this sample of persons with serious mental illnesses. This suggests that motivations for CAM use may vary by population and condition. Because few correlates of CAM use among persons with serious mental illnesses are known, providers should conduct routine assessments of CAM use.
\end{abstract}

\section{Introduction}

C OMPLEMENTARY AND ALTERNATIVE MEDICINE (CAM) refers to a variety of diverse treatment practices that are not considered conventional medicine. Complementary medicine refers to therapies used in combination with conventional therapies, while alternative medicine refers to therapies used instead of conventional medicine. ${ }^{1}$ Although relatively little research has examined the use of CAM among adults with serious mental illnesses (SMI), interest in the topic is increasing. Recent studies suggest that rates of CAM use among this group are relatively high. ${ }^{2,3}$ For example, a recent survey found that $22 \%$ of adults with mania or psychosis used some form of CAM therapy. 4

Although interest in CAM is growing, very little is known about the patterns and correlates of use among persons with serious mental illnesses. This is an important area of investigation, as clinicians need to be aware of potential interactions with conventional treatments. ${ }^{5}$ Provider awareness of their patient's use of CAM therapies may be important to ensuring that patient preferences are recognized to help ensure continued engagement in conventional treatment. In

\footnotetext{
${ }^{1}$ School of Social Work, The University of Michigan, Ann Arbor, MI. MI.

${ }^{2}$ Department of Veterans Affairs National Serious Mental Illness Treatment and Research Education Center (VA SMITREC), Ann Arbor,

${ }^{3}$ RAND Corporation, Pittsburgh, PA.
} 
particular, it is important to understand how often and for what reasons persons with SMI use CAM therapies. Historically, the quality of conventional treatment for persons with SMI has been poor. ${ }^{6,7}$ Persistent distress due to symptoms of mental illness and perceived ineffectiveness of treatment may motivate patients to seek relief through other means, including CAM therapies.

Use of CAM therapies may also be associated with medication nonadherence. Recent pharmacologic agents still have significant side-effects including weight gain, nausea, diarrhea, sedation, anxiety, and others, ${ }^{8}$ and nonadherence to medications for mental disorders remains high. For example, more than one third of patients with bipolar disorder have reported rates of nonadherence as high as $60 \% .^{9,10}$ Persons who are nonadherent may be more likely to experiment with or regularly use CAM therapies to relieve symptoms, especially if they feel that their overall treatment regimen is ineffective. Complicating factors such as drug-drug interactions, drug substitutions with CAM, and treatment noncompliance demand a greater understanding of factors associated with CAM use among persons with serious mental illnesses.

This study tested whether CAM use was associated with perceived effectiveness of conventional treatment and medication compliance among a large sample of persons with bipolar disorder. Bipolar disorder is an ideal sample for this investigation due to the chronicity of the condition, public burden, ${ }^{7}$ significant medication side-effects, ${ }^{11}$ and documented high rates of CAM use among this population. ${ }^{2,3,11}$

\section{Materials and Methods}

\section{Participants}

Participants were recruited from the Continuous Improvement for Veterans in Care-Mood Disorders (CIVIC-MD) study. ${ }^{12}$ Details about this study are available elsewhere. ${ }^{12}$ Briefly, the CIVIC-MD is a naturalistic cohort study of 435 veterans diagnosed with bipolar disorder recruited from a large urban Veterans Affairs (VA) mental health facility in the mid-Atlantic region. Data were collected from July 2004 to July 2006. Seventy-seven percent (77\%) of subjects $(n=336)$ were white, and $13 \%(n=58)$ were black. Fourteen percent $(14 \%)$ of subjects $(n=62)$ were women. Average incomes were low. Sixty percent $(60 \%)(n=254)$ earned less than $\$ 20,000$ per year. The average age of subjects was 49 years $(S D=11)$, with a range of $21-78$ years. Additional details regarding the CIVIC-MD study are available elsewhere. $^{12}$

Inclusion criteria included a current diagnosis of bipolar disorder (I, II, NOS), cyclothymia, or schizoaffective disorder-bipolar subtype based upon chart review and provider confirmation.

The study was reviewed and approved by the Veterans Affairs Institutional Review Board. Seven hundred and twenty (720) eligible veterans were approached for participation, and 435 completed the self-administered survey. Exclusions occurred for the following reasons: the patient refused to participate $(n=148)$, the provider determined that the patient was not capable of providing consent $(n=104)$, or the patient enrolled but did not complete the survey $(n=33)$. The effective sample size for the current study after exclusion of missing data was $N=429$. Logistic regression was used to examine patterns of missing data and none were identified.

\section{Measurement}

Treatment effectiveness. Perceived effectiveness of psychotropic medications and psychotherapy were assessed using items adapted from Meredith and colleagues. ${ }^{13}$ Questions elicited veterans' thoughts about whether medications and psychotherapy relieved bipolar symptoms, improved functioning, minimized side-effects, and prevented symptomatic relapse. Four (4) items measured perceived effectiveness of medications, and four parallel items measured effectiveness of psychotherapy. Responses ranged from 0 ("very unlikely") to 4 ("very likely").

Medication compliance. Medication compliance was assessed using the validated Morisky scale, ${ }^{14}$ a four-item yes/ no instrument frequently used for assessing patient adherence to treatment across a variety of chronic medical and psychiatric conditions, including affective disorders. ${ }^{15,16}$ The Morisky scale includes items querying whether the patient ever forgot to take medications, was careless with medications, at times stopped taking medications when feeling better, or stopped taking medications when feeling worse. The four items were summed, with higher scores reflecting greater levels of compliance. Recent research has shown this scale to have good reliability $(\alpha=.83)$, and concurrent and predictive validity in outpatient settings. ${ }^{14,17}$

CAM utilization. CAM utilization was based on a previously established questionnaire by Kessler et al. ${ }^{18}$ This survey was selected because it included a wide range of CAM therapies observed in patients with bipolar disorder in prior studies, in addition to the broader population. ${ }^{4}$ Participants were asked whether they used any of 14 therapies within the past year (yes/no). Four (4) categories of CAM therapies were considered based on previously established definitions by Kessler et al. ${ }^{16}$ : physical, oral medications, cognitive, and dietary. Physical CAM included use of acupuncture/ acupressure, chiropractic, or massage therapies. Oral-based CAM included herbal medications, homeopathy, St. John's Wort, or vitamins/minerals. Cognitive-based CAM included relaxation/breathing exercises, imagery, or meditation. Dietoriented CAM included dietary/weight loss supplements or any special dietary modifications. Spirituality and self-help practices were among the 14 therapies assessed. Because they are widely recognized as a standard of care among populations seeking treatment for SMI, they were not included in this study of CAM.

Sociodemographics. Sociodemographic variables were examined in this study were age, gender, marital status, education, and employment status.

Statistical analysis. Data were summarized using descriptive statistics. Associations between CAM use and perceived effectiveness of conventional therapies and medication adherence were examined using $t$ tests. Statistically significant bivariate associations were tested using multivariate linear regression models, which controlled for potentially confounding sociodemographic characteristics. Statistically 
significant bivariate associations were examined with linear regression models, controlling for potentially confounding sociodemographic characteristics. Sociodemographic variables included marital status, gender, employment status, age, and education level. In analyzing statistically significant associations, we used logistic regression for categorical data and multivariate linear regression elsewhere. While not mutually exclusive, CAM categories were analyzed independently. Thus, it is possible that participants used more than one form of CAM.

\section{Results}

\section{Perceived effectiveness of medications}

Table 1 provides a summary of bivariate associations between CAM use and perceived effectiveness of treatment. Patients who used oral or cognitive CAM were more likely to indicate that their medications were not effective at relieving manic or depressive symptoms $(t[424.6]=0.65, p=0.034$; $t[419.2]=2.39, p=0.017$, respectively). Users of cognitive CAM were also more likely to report that their medications did not help with social, job, or family functioning $(t[412.6]=2.10, p=0.036)$, and that their medications did not prevent recurrences of manic or depressive episodes $(t[423.6]=2.16, p=0.031)$. Users of physical and diet CAM therapies did not exhibit differences in their perceived effectiveness of medications. The multivariate linear regression models that tested the association between CAM use (i.e., oral and cognitive) and perceived effectiveness of medications while controlling for sociodemographic factors was nonsignificant $(p>0.05)$.

\section{Perceived effectiveness of psychotherapy}

Regarding perceived effectiveness of psychotherapy, only one significant association was observed. Patients who used cognitive CAM were more likely to report that psychotherapy was not effective at improving social, family, or job functioning $(t=2.265, d f=412.3, p=0.024)$. The multivariate linear regression model that tested the association between cognitive CAM use and perceived effectiveness of psychotherapy was nonsignificant $(p>0.05)$.

\section{Medication adherence}

Medication adherence was not associated with any type of CAM use $(p>0.05)$. Associations were tested using individual items of the Morisky scale and the overall scale score.

\section{Discussion}

Although many patients use CAM therapies for mental disorders, including SMI, very little is known about factors associated with use of CAM. To our knowledge, this is the first study to examine CAM use and its associations with perceived effectiveness of conventional treatment and medication compliance. Overall, we found some evidence that persons who used CAM were slightly more likely to report that their medications were ineffective. Use of oral and cognitive CAM modestly predicted perceptions that medications did not relieve mania or depression. Cognitive CAM uniquely predicted perceptions that medications did not relieve social difficulties or prevent relapses of mania or de- pression. However, these associations were of a small effect size and were not significant after controlling for other sociodemographic factors.

These findings are not consistent with the broader literature on CAM use for general medical conditions, in particular, findings that suggest that use of CAM may be sought because conventional treatments are not perceived as effective. ${ }^{18,19}$ Some potential explanations are offered for these differential findings. One possibility is that participants in this survey may be using CAM for additional symptoms beyond their existing conventional treatment, which is considered generally effective. Subsequent research using qualitative methods to understand reasons for using CAM would be helpful, as this would help clarify the range of motivations and belief structures about CAM. Qualitative methods could also shed light on an important distinction, namely, whether participants are using CAM therapies as a complement or alternative to conventional medicine, or both. In addition, their rationales for incorporating specific therapies could be explored.

Another potential explanation relates to the study design. CAM was measured as a lifetime variable, while frequency and timing of CAM use were not considered. Patients may have experimented with various types of CAM therapies over time, which would attenuate any real associations. Additionally, a selection bias in this sample may be present. That is, persons who find their treatment inadequate may have dropped out prior to this study, and we are unaware of CAM use within this group. Improvements in measurement and sampling design will be necessary to advance this area of research.

Finally, study participants may be using CAM to improve overall health and not simply in response to specific symptoms that are targeted by psychotherapy or psychotropic medications. Thus, patients may not consider these therapies to be effective for specific symptom relief but rather for general well-being that is not influenced by conventional treatment. Indeed, prior research indicates that patients often use many forms of CAM to improve well-being rather than as a targeted treatment for specific symptoms. ${ }^{5}$

Strengths of this study include the large sample size and a population actively seeking treatment for bipolar disorder. The use of validated measures of medication compliance and CAM use are also beneficial. Study findings should also be considered in the context of the limitations. Most notably, subjects in this study were recruited from a VA clinic that serves patients with relatively severe symptoms. The sample is over-represented by older men who tend to earn less and have lower educational levels than subjects in representative national samples. Thus, the findings may not generalize to other clinic settings or the general population.

\section{Conclusions}

Overall, this study provides evidence that CAM use is not associated with perceived effectiveness of treatment. Unlike the general population of CAM users, the research to date has not revealed any consistent patterns of CAM use among persons with serious mental illnesses. Given the high rate of CAM use among persons with SMI, it is important that providers adopt a person-centered approach with respect to CAM: that is, assessing for its use and respecting that CAM 


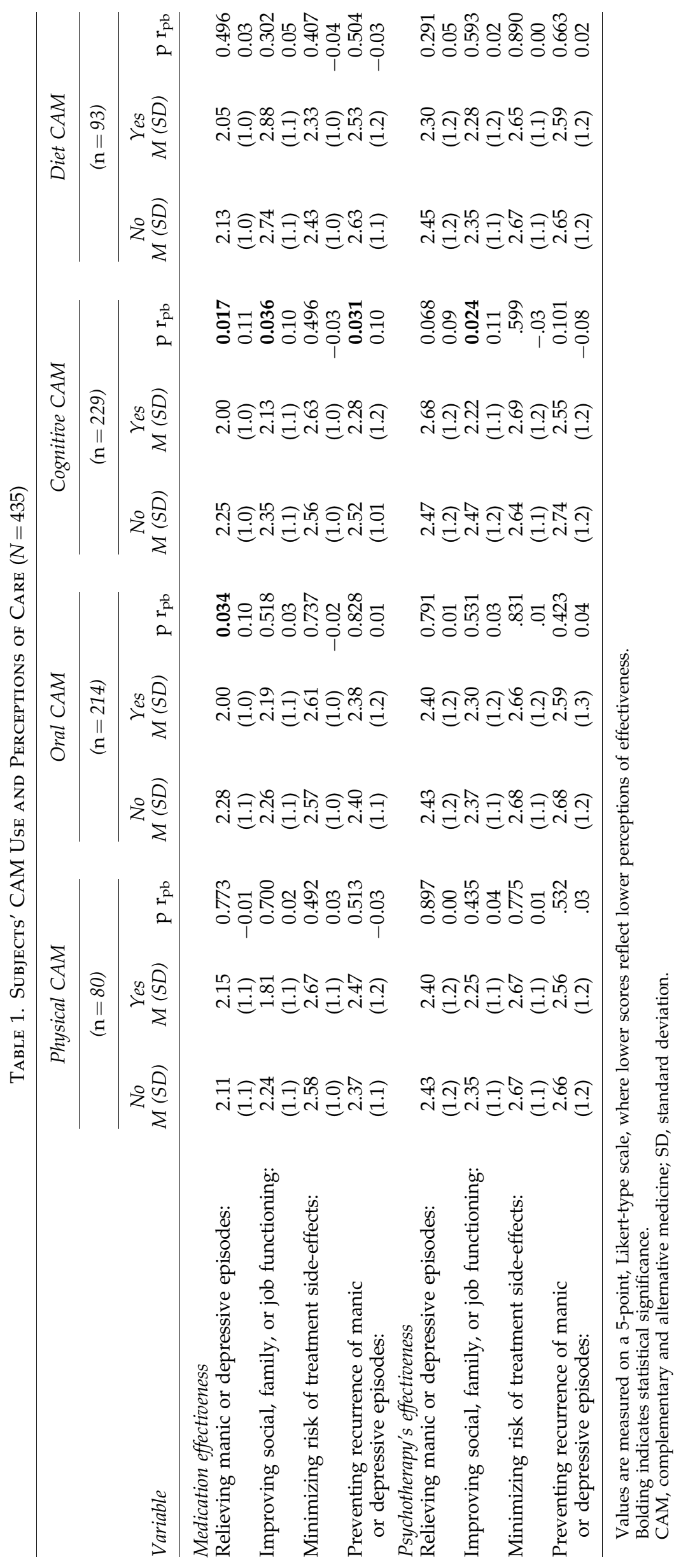


may be an important part of the patient's treatment preferences. Furthermore, disclosure of CAM use is low even among adults with chronic conditions. ${ }^{20}$ Engaging in this discussion is particularly important, given the potential for drug interactions. ${ }^{5,21}$

\section{Acknowledgments}

This research was supported by the Department of Veterans Affairs, Veterans Health Administration, Health Services Research and Development Service and Office of Mental Health Services, and the Curtis Center of the University of Michigan, School of Social Work. The views expressed in this article are those of the authors and do not necessarily represent the views of the Department of Veterans Affairs or the Curtis Center.

\section{Disclosure Statement}

No competing financial interests exist.

\section{References}

1. Tindle HA, et al. Trends in use of complementary and alternative medicine by US adults: 1997-2002. Altern Ther Health Med 2005;11:42-49.

2. Kilbourne AM, et al. Determinants of complementary and alternative medicine use by patients with bipolar disorder. Psychopharmacol Bull 2007;40:104-115.

3. Dennehy EB, Gonzalez R, Suppes T. Self-reported participation in nonpharmacologic treatments for bipolar disorder. J Clin Psychiatry 2004;65:278.

4. Unutzer J, et al. Mental disorders and the use of alternative medicine: Results from a national survey. Am J Psychiatry 2000;157:1851-1857.

5. Andreescu C, Mulsant BH, Emanuel JE. Complementary and alternative medicine in the treatment of bipolar disorder: A review of the evidence. J Affect Disord 2008;110:16-26.

6. Lehman AF, Steinwachs DM. Patterns of usual care for schizophrenia: Initial results from the Schizophrenia Patient Outcomes Research Team (PORT) Client Survey. Schizophr Bull 1998;24:11-20; discussion 20-32.

7. Merikangas KR, et al. Lifetime and 12-month prevalence of bipolar spectrum disorder in the National Comorbidity Survey replication. Arch Gen Psychiatry 2007;64:543-552.

8. Manwani SG, et al. Adherence to pharmacotherapy in bipolar disorder patients with and without co-occurring substance use disorders. J Clin Psychiatry 2007;68:1172-1176.

9. Scott J, Pope M. Nonadherence with mood stabilizers: Prevalence and predictors. J Clin Psychiatry 2002;63:384-390.
10. Greenhouse WJ, Meyer B, Johnson SL. Coping and medication adherence in bipolar disorder. I Affect Disord 2000;59: 237-241.

11. Baldessarini RJ, Perry R, Pike J. Factors associated with treatment nonadherence among US bipolar disorder patients. Hum Psychopharmacol 2008;23:95-105.

12. Kilbourne AM, et al. The continuous improvement for veterans in care: Mood Disorders (civic-md) Study, a VAacademic partnership. Psychiatr Serv 2008;59:483-485.

13. Meredith LS, et al. Treating depression in staff-model versus network-model managed care organizations. I Gen Intern Med 1999;14:39-48.

14. Morisky DE, et al. Predictive validity of a medication adherence measure in an outpatient setting. J Clin Hypertens (Greenwich) 2008;10:348-354.

15. Miklowitz DJ, et al. Family-focused treatment of bipolar disorder: 1-year effects of a psychoeducational program in conjunction with pharmacotherapy. Biol Psychiatry 2000;48: 582-592.

16. Shalansky SJ, Levy AR, Ignaszewski AP. Self-reported Morisky score for identifying nonadherence with cardiovascular medications. Ann Pharmacother 2004;38:13631368.

17. Morisky DE, Green LW, Levine DM. Concurrent and predictive validity of a self-reported measure of medication adherence. Med Care 1986;24:67-74.

18. Kessler RC, et al. The use of complementary and alternative therapies to treat anxiety and depression in the United States. Am J Psychiatry 2001;158:289-294.

19. Astin JA. Why patients use alternative medicine: Results of a national study. JAMA 1998;279:1548-1553.

20. Mehta DH, Gardiner PM, Phillips RS, McCarthy EP. Herbal and dietary supplement disclosure to health care providers by individuals with chronic conditions. I Altern Complement Med 2008;14:1263-1269.

21. Markowitz JS, DeVane CL. The emerging recognition of herb-drug interactions with a focus on St. John's wort (Hypericum perforatum). Psychopharmacol Bull 2001;35: 53-64.
Address correspondence to: Brian E. Perron, Ph.D., L.C.S.W. School of Social Work University of Michigan 1080 South University Avenue Ann Arbor, MI 48109

E-mail: beperron@umich.edu 

This article has been cited by:

1. 2010. RECENT LITERATURE. Focus on Alternative and Complementary Therapies 15:3, 264-269. [CrossRef] 